\title{
Comparing surgical trays with redundant instruments with trays with reduced instruments: a cost analysis
}

\author{
A. John-Baptiste PhD, L.J. Sowerby MD MHM, C.J. Chin MD, J. Martin PharmD MSc, \\ B.W. Rotenberg MD MPH
}

Abstract

Background: When prearranged standard surgical trays contain instruments that are repeatedly unused, the redundancy can result in unnecessary health care costs. Our objective was to estimate potential savings by performing an economic evaluation comparing the cost of surgical trays with redundant instruments with surgical trays with reduced instruments ("reduced trays").

Methods: We performed a cost-analysis from the hospital perspective over a 1-year period. Using a mathematical model, we compared the direct costs of trays containing redundant instruments to reduced trays for 5 otolaryngology procedures. We incorporated data from several sources including local hospital data on surgical volume, the number of instruments on redundant and reduced trays, wages of personnel and time required to pack instruments. From the literature, we incorporated instrument depreciation costs and the time required to decontaminate an instrument. We performed 1-way sensitivity analyses on all variables, including surgical volume. Costs were estimated in 2013 Canadian dollars.

Results: The cost of redundant trays was $\$ 21806$ and the cost of reduced trays was $\$ 8803$, for a 1 -year cost saving of $\$ 13003$. In sensitivity analyses, cost savings ranged from $\$ 3262$ to $\$ 21395$, based on the surgical volume at the institution. Variation in surgical volume resulted in a wider range of estimates, with a minimum of $\$ 3253$ for low-volume to a maximum of $\$ 52012$ for high-volume institutions.

Interpretation: Our study suggests moderate savings may be achieved by reducing surgical tray redundancy and, if applied to other surgical specialties, may result in savings to Canadian health care systems.

\begin{abstract}
n 2015, total health care expenditures in Canada were estimated at $\$ 219$ billion. $^{1}$ Although hospitals represented the largest category of expenditures at $29.5 \%$, this proportion has been steadily decreasing over the last 2 decades. The decline is due in part to provincial and territorial policies to promote cost cutting in hospitals. In Ontario, activity-based funding in the form of Health-Based Allocation Models has been implemented to promote quality care and incentivize increased efficiency. ${ }^{2,3}$ Similar funding initiatives have previously been implemented in British Columbia and Alberta. ${ }^{4}$ As a result, hospitals are keen to identify areas of potential cost savings.

Surgical tray redundancy is recognized as a difficulty in surgical units. ${ }^{5-8}$ At one hospital, a review of 49 procedures and 247 trays within 4 surgical specialties (otolaryngology, plastic surgery, bariatric surgery and neurosurgery) showed that rates of instrument use varied from $13.0 \%$ to $21.9 \% .^{7}$ After surgery, sterile processing personnel decontaminate instruments through manual cleaning. Personnel assemble standardized trays by packing instruments onto trays, which are then washed and sterilized in a washer-disinfector
\end{abstract}

machine for the next surgical procedure. Since all instruments in an opened tray require sterile processing, unused instruments incur potentially avoidable costs. In a previous study, we conducted a review of instruments on surgical trays in the otolaryngology departments of St. Joseph's Health Care London and the London Health Sciences Centre, London, Ont. ${ }^{9}$ We found that the average tray use ranged from $20.1 \%$ to $51.7 \%$, suggesting substantial redundancy. We have proposed streamlined trays - "reduced trays" — that would reduce the number of instruments by more than $50 \%$. In this study, our objective was to perform an economic evaluation of streamlined trays to quantify the potential cost savings that may result from implementing the reduced trays.

Competing interests: None declared.

This article has been peer reviewed.

Correspondence to: A. John-Baptiste, ajohnbap@uwo.ca

CMAJ Open 2016. DOI:10.9778/cmajo.20150092 


\section{Methods}

We conducted a model-based economic evaluation comparing redundant trays to reduced trays. We performed a costanalysis for 5 common otolaryngology surgical procedures (septoplasty, septorhinoplasty, skin cancer excision, endoscopic sinus surgery and tonsillectomy). The analysis was performed from the hospital perspective and incorporates the costs incurred by the hospital in sterile processing of surgical trays. The model estimated costs as a function of the number of instruments on the tray, the number of surgical procedures, the per instrument decontamination and packing times, personnel time costs and the per instrument depreciation cost.

$\operatorname{Cost}_{\text {tray }}=\left(n_{\text {procedures }} \times n_{\text {instruments }}\right) \times\left[\operatorname{cost}_{\text {time }}\left(\right.\right.$ time $_{\text {decontaminate }}+$ time $\left._{\text {pack }}\right)+$ cost $\left.t_{\text {depreciation }}\right)$

Costs were estimated in 2013 Canadian dollars.

\section{Data sources}

We incorporated data from several sources into the model. We used local hospital data from 2 tertiary care academic hospitals, the London Health Sciences Centre and St. Joseph's Health Care, in London, Ont. For the 5 otolaryngology procedures, we obtained 2013 hospital data on surgical volumes. We incorporated data on the number and composition of extant trays and proposed reduced trays comprising fewer, more frequently used instruments, based on findings from our earlier review of tray use. ${ }^{9}$ The details of extant and reduced tray composition are provided in Appendix 1 (available at www.cmajopen.ca/content/4/3/E404/suppl/DC1).

To estimate the time to pack instruments during tray assembly, we obtained routinely collected data from the hospital central processing unit at the London Health Sciences Centre on the time to assemble otolaryngology trays between Jan. 1, 2013, and Dec. 31, 2013. The data set represented 173 trays, with a total of 9445 assemblies, and provided the mean packing time per tray across all surgical procedures. To obtain a sample representative of the 5 tray categories of interest, we selected tray categories with a minimum of 10 instruments, and a minimum of 10 repeated assemblies. For each tray, we divided the total number of instruments per tray by the average tray assembly time to calculate a mean per instrument packing time and incorporated the 2.5 and 97.5 percentiles of the per instrument packing time as the extreme ends of the range for sensitivity analysis. From the human resources department, we obtained the mean wage rates of central processing personnel, along with the minimum and maximum wage rates. We calculated a per second wage rate by dividing hourly rates by 3600 .

Because routinely collected data on decontamination times and per instrument depreciation costs were not available from the local hospitals, we conducted a review of the literature to identify studies with relevant information. We incorporated a minimum and maximum decontamination time for trays with more than 10 instruments from the University of Chicago Medicine Hospitals study using data from 61 trays. $^{7}$ From the literature, we incorporated a mean per instrument depreciation cost of US\$0.06 (minimum US\$0.02, maximum US\$0.18), which had been calculated by dividing instrument purchase prices by their estimated lifespans. ${ }^{7} \mathrm{We}$ used the purchasing power parity for health of 1.0206 to convert US dollars to Canadian dollars. ${ }^{10}$ The purchasing power parity for health is an exchange rate estimated by comparing the price of a standard package of health-related goods and services, including hospital services, between 2 countries.

\section{Statistical analyses}

To compare our estimates with others from the literature, we estimated a per instrument labour cost by adding the per instrument decontamination and packing times and multiplying by the per second labour cost. ${ }^{7}$ All analyses were performed using $\mathrm{R}$ for Statistical Computing ( $\mathrm{R}$ Foundation for Statistical Computing, Vienna, Austria; https://www .R-project.org/).

\section{Sensitivity analyses}

We performed a series of one-way sensitivity analyses. We varied each parameter across its plausible range, with the other parameters set to their base case values. In this way, we characterized minimum and maximum cost savings for the range of each input parameter. We varied the reduction in tray size from $25 \%$ to $110 \%$, corresponding to less reduction in tray size and greater reduction, respectively. We also, varied the surgical volume between $25 \%$ and $400 \%$, corresponding to a low and high surgical volume, respectively. The surgical volume at our institution does not vary to a great extent. Sensitivity analysis on surgical volume is, however, pertinent to the external generalizability of our estimates to other institutions.

\section{Scenario analysis}

In the study conducted at the University of Chicago Medicine Hospitals, investigators estimated indirect cost savings from reduced trays for 49 procedures, spanning 4 surgical services (otolaryngology, plastic surgery, bariatric surgery and neurology). Indirect cost savings were estimated by allocating the operating costs of the sterile processing unit to each instrument to account for decreases in utilities, reagents, quality checks and equipment maintenance. ${ }^{7} \mathrm{We}$ incorporated these additional cost savings into a scenario analysis, rather than the base case analysis, owing to concerns that reductions in indirect cost are sensitive to scale. In our hospital, reagents for the washer-disinfector are used in standard aliquots irrespective of the number of surgical trays or the number of instruments in the machine. Reducing trays for 5 surgical procedures would not likely result in fewer cycles of operation for the washer-disinfector, and thus substantial savings from utilities, reagents and equipment repair and maintenance would not likely be realized.

\section{Results}

In the base case analysis, the estimated annual cost of redundant trays at London Health Science Centre and St. Joseph's 


\section{OPEN}

Research

Health Care for the 5 procedures was \$21 806 and the annual cost of reduced trays was expected to be $\$ 8803$. Our analysis suggests that if we had implemented the proposed tray reduction at our institution, based on the number of procedures performed in 2013, we may have saved $\$ 13003$ in that year. Our base case per instrument labour cost was $\$ 0.13$.

\section{Study parameters}

The 2013 hospital data on surgical volumes are shown in Table 1. Of a total of 173 trays, 39 met our inclusion criteria, representing 4541 assemblies. We calculated a mean per instrument packing time of $17.5 \mathrm{~s}$ and a per second cost of personnel time of $\$ 0.006$ (Table 2). We incorporated a decontamination time of $4.02 \mathrm{~s}$ per instrument. Minimum and maximum decontamination times were $1.07 \mathrm{~s}(15 \mathrm{~s}, 14$ instruments) and 13.64 s (22 s, 300 instruments) per instrument, respectively. In the scenario analysis, we incorporated a per instrument indirect cost of US\$0.23 by converting to Canadian dollars using the purchasing power parity (Table 2). ${ }^{7,10}$

\section{Sensitivity analysis}

Based on the surgical volume at our own institution, the extent of tray reduction was an important factor in the costsaving estimates. One-way sensitivity analysis suggested that removing fewer instruments from the tray at $25 \%$ of the base case reduction would result in a cost saving of $\$ 3262$, whereas

Table 1: Annual surgical volumes at London Health Sciences Centre and St. Joseph's Health Care

\begin{tabular}{|lccccc|}
\hline Procedure & $\begin{array}{c}\text { No. of procedures } \\
\text { each year }\end{array}$ & $\begin{array}{c}\text { No. of instruments } \\
\text { on extant trays }\end{array}$ & $\begin{array}{c}\text { No. of instruments } \\
\text { on proposed trays }\end{array}$ & Difference & Percent reduction \\
\hline Septoplasty & 197 & 84 & 33 & 51 & 61 \\
\hline Tonsillectomy & 336 & 34 & 13 & 21 & 62 \\
\hline Skin cancer excision & 220 & 43 & 27 & 36 & 64 \\
\hline Endoscopic sinus surgery & 505 & 100 & 60 & 82 & 64 \\
\hline Septorhinoplasty & 190 & 142 & & & 58 \\
\hline
\end{tabular}

Table 2: Model input parameters

\begin{tabular}{|lccl|}
\hline Variables & $\begin{array}{c}\text { Base case } \\
\text { value }\end{array}$ & Range & \multicolumn{1}{c|}{ Source } \\
\hline Per instrument decontamination time, s & 4.02 & $1.07-13.64$ & Stockert and colleagues $^{7}$ \\
\hline Per instrument packing time, s & 17.5 & $7.6-31.6$ & Analysis of local hospital data on tray packing times \\
\hline Per second cost of personnel time, \$ & 0.006 & $0.0058-0.0061$ & $\begin{array}{l}\text { Derived from hospital human resources data by dividing } \\
\text { hourly wages by } 3600\end{array}$ \\
\hline Per instrument cost of depreciation, \$ & 0.06 & $0.02-0.18$ & $\begin{array}{l}\text { Derived from Stockert and colleagues; }{ }^{7} \text { purchasing power } \\
\text { parities for health }\end{array}$ \\
\hline Per instrument indirect cost, \$ & 0.23 & NA & $\begin{array}{l}\text { Derived from Stockert and colleagues; }{ }^{7} \text { purchasing power } \\
\text { parities for health }\end{array}$ \\
\hline
\end{tabular}

Table 3: Results of 1-way sensitivity analysis

\begin{tabular}{|c|c|c|c|c|}
\hline Variable & Minimum value & Maximum value & $\begin{array}{l}\text { Minimum cost } \\
\text { saving, } \$\end{array}$ & $\begin{array}{l}\text { Maximum cost } \\
\text { saving, } \$\end{array}$ \\
\hline Per instrument decontamination time, s & 1.07 & 13.64 & 11796 & 16940 \\
\hline Per second cost of personnel time, $\$$ & 0.0058 & 0.0061 & 12798 & 13208 \\
\hline Per instrument cost of depreciation, \$ & 0.02 & 0.18 & 10205 & 21395 \\
\hline Surgical volume & $\begin{array}{l}\text { Lower surgical volume } \\
\text { ( } 25 \% \text { of base case) }\end{array}$ & $\begin{array}{l}\text { Higher surgical volume } \\
\text { ( } 400 \% \text { of base case) }\end{array}$ & 3253 & 52012 \\
\hline
\end{tabular}


removing $10 \%$ more than the base case would result in a cost saving of $\$ 14300$ (Table 3). The per instrument packing time was also an important factor. In one-way sensitivity analysis, the estimated cost savings ranged from $\$ 8951$ to $\$ 18773$ over the plausible range of per instrument packing times (Table 3).

Variation in surgical volume was associated with the largest variation in cost savings estimates. With all other parameters remaining the same, an institution performing $1 / 4$ of the volume would save $\$ 3253$ (Table 3). An institution performing 4 times the surgical volume would save $\$ 52012$ per year (Table 3).

\section{Scenario analysis}

In a scenario analysis that incorporated indirect cost savings, the cost of redundant trays was $\$ 48781$, the cost of reduced trays $\$ 19692$ and the estimated cost savings was $\$ 29088$ per year.

\section{Interpretation}

Our analysis suggests cost savings can be achieved by reducing surgical trays for 5 otolaryngology procedures. Although these savings are modest when considered in the context of the entire hospital budget, the effect could be greater if redundancy were addressed for a broader range of surgical procedures across specialties.

Our per instrument labour cost of $\$ 0.13$ is comparable with the US\$0.10 per instrument estimated by Stockert and colleagues. ${ }^{7}$ Our institution did have a higher per instrument packing time of 17.6 seconds indicated by our local hospital data, compared with 12.51 seconds in the previous study. ${ }^{7}$ Our per instrument cost is lower than that obtained by Morris and colleagues, with a per instrument processing cost of US $\$ 0.70$, and lower that of than Florijn, with an estimate of $€ 1$ per instrument. ${ }^{5,11}$ The input data for these cost estimates are not provided and thus the reasons for the higher costs when compared with our study are unclear. Our per instrument processing cost was also lower than that of Farrokhi and colleagues, with a per instrument processing cost of US $\$ 0.77 . .^{12}$ Their estimate incorporated indirect costs, which accounts for some of the discrepancy when compared with our per instrument cost.

Several studies reported overall cost-saving estimates. In the Netherlands, cost savings were estimated to range between $€ 55000$ and $€ 81360$ each year. ${ }^{5}$ Farrokhi and colleagues estimated potential savings of up to $\$ 2.8$ million a year, with a $70 \%$ reduction in instrument processing for all surgical procedures at Virginia Mason Medical Center, a 300bed hospital with 24 operating rooms. ${ }^{12}$ The higher cost saving estimates when compared with our base case estimate are due in large part to the inclusion of a broader range of surgical procedures, ${ }^{5,12}$ and inclusion of overhead costs. ${ }^{12}$ Wannemuehler and colleagues estimated annual savings of $\$ 850$ in sterile processing costs and a further $\$ 1468.99$ savings in instrument purchasing costs associated with reducing an adenotonsillectomy tray. ${ }^{8}$

Indirect cost savings have been incorporated into other surgical instrument cost analyses., ${ }^{72,13}$ Our base case estimate of savings conservatively excluded overhead costs. Our cost estimate would be an underestimate of the overall savings if the reduction in 5 otolaryngology trays were applied within a strategy spanning multiple procedures and surgical services. Our scenario analysis, incorporating the indirect costs of reagents, utilities, quality checks and equipment maintenance, suggested higher potential cost savings of \$29088.

\section{Limitations}

Our study is model based and, therefore, we incorporated data from a study conducted in the US. Uncertainty about variables can affect the results and lead to over or underestimation of the savings. We mitigated this effect by incorporating local data when possible, converting from US dollars to Canadian dollars using purchasing power parities and performing extensive sensitivity analysis.

Our study did not account for effects on operating room processes. Tray reduction may result in faster operating room set up, easier retrieval of instruments during operations and faster operating room clean-up. The evidence suggests that tray reduction speeds up operating room set up. This has recently been shown in the otolaryngology literature, with a significant 1-minute reduction in operating room turnover before and after reducing the number of instruments on the adenotonsillectomy tray. ${ }^{8}$ Farrokhi and colleagues compared processes before and after tray optimization and found that a $70 \%$ reduction in the number of instruments for minimally invasive spine surgery (197 to 58), decreased set up time by $37 \%$ (from 13.1 to $8.2 \mathrm{~min}, p=0.0015$ ). ${ }^{12}$

Tray reduction may also result in adverse consequences such as the need, albeit infrequently, to retrieve instruments that are not on the surgical trays during procedures. Making a tray readily accessible in the operating room to be opened when extra instruments are required is a precautionary measure that can ensure clinical outcomes are not adversely affected. After implementing reduction in the adenotonsillectomy tray, Wannemuehler and colleagues found that the extra tray was accessed $3.6 \%$ of the time and that $93.75 \%$ of surgeons and other personnel were satisfied with the reductions. ${ }^{8}$ An extra tray would attenuate tray reduction cost savings slightly, owing to the need for sterile processing of the extra tray.

The cost savings we estimated may not represent real savings to the hospital, particularly if there are no adjustments to the number of hours worked by central processing personnel. However, we are confident in our assumption of a linear relationship between time savings and cost savings. At our institutions, central processing personnel are paid by the hour, and managers have recently reduced hours to address budgetary challenges. In 2015, the reduction in employee hours at London Health Sciences Centre was equivalent to 97 full-time positions and was equivalent to 24 full-time positions at St. Joseph's Health Care. ${ }^{14}$ This suggests that time savings from reduced trays have the potential to translate into cost savings.

The cost savings are based on a sampling of retrospective data, and ideally would be validated through prospective implementation of tray optimization. 


\section{Conclusion}

Eliminating tray redundancy for 5 otolaryngology procedures is associated with a potential cost saving of \$13 003. Owing to the promise for cost savings, the extent and generalizability of the savings, in addition to the impact on operating room processes, should be explored further in prospective studies. If tray redundancy is as common as literature-based estimates suggest, the broader implications for health care systems in Canada would be substantial when projected over a number of surgical procedures. For hospitals and departments interested in tray reduction, our suggestion would be to engage all invested parties, including nurses, physicians and operating room personnel, to identify frequently opened trays and to measure instrument utilization rates. Commercial products exist that can help facilitate instrument reduction and supply chain optimization. Our findings indicate that eliminating tray redundancy may be a simple and feasible opportunity for hospitals seeking to reduce costs.

\section{References}

1. National health expenditure trends, 1975 to 2015. Ottawa: Canadian Institute for Health Information; 2015. Available: https://secure.cihi.ca/free_products /nhex_trends_narrative_report_2015_en.pdf (accessed 2016 Mar. 5).

2. Health System Funding Reform (HSFR). Toronto: Ontario Ministry of Health and Long-Term Care; 2015. Available: www.health.gov.on.ca/en/ pro/programs/ecfa/funding/hs_funding.aspx (accessed 2015 July 20).

3. Sutherland JM. Hospital payment mechanisms: an overview and options for Canada. Ottawa: Canadian Health Services Research Foundation; 2011. Available: www.cfhi-fcass.ca/Libraries/Hospital_Funding_docs/CHSRF-SutherlandHospitalFundingENG.sflb.ashx (accessed 2015 July 4).

4. Surgical volume trends, 2008: within and beyond wait time priority areas. Ottawa: Canadian Institute for Health Information; 2008

5. Kroes L. Creating more efficiency and patient safety by changing processes and contents of instrument trays. M.Sc, thesis, Health Science School of Management and Governance, Department of Science, Technology, Health and Policy studies (STeHPS) University of Twente, Enschede, The Netherlands; 2009.

6. Morris LF, Romero Arenas MA, Cerny J, et al. Streamlining variability in hospital charges for standard thyroidectomy: Developing a strategy to decrease waste. Surgery 2014;156:1441-9, discussion 1449.
7. Stockert EW, Langerman A. Assessing the magnitude and costs of intraoperative inefficiencies attributable to surgical instrument trays. 7 Am Coll Surg 2014;219:646-55.

8. Wannemuehler TJ, Elghouche AN, Kokoska MS, et al. Impact of Lean on surgical instrument reduction: Less is more. Laryngoscope 2015;125:2810-5.

9. Chin CJ, Sowerby LJ, John-Baptiste A, et al. Reducing otolaryngology surgical inefficiency via assessment of tray redundancy. 7 Otolaryngol Head Neck Surg 2014;43:46.

10. Choosing interventions that are cost effective (WHO-CHOICE): Purchasing Power Parity 2005. Geneva: World Health Organization: 2005. Available: www.who.int/choice/costs/ppp/en/ (accessed 2015 July 20).

11. Florijn EP. Optimisation of the distribution of surgical instruments over trays. Cost effectiveness and quality improvement of an operating theatre. M.Sc. thesis, Industrial Engineering and Management, Logistical Processes in Healthcare, University of Twente, Enschede, The Netherlands; 2008.

12. Farrokhi FR, Gunther M, Williams B, et al. Application of lean methodology for improved quality and efficiency in operating room instrument availability. 7 Healthc Qual 2015;37:277-86.

13. Prat F, Spieler JF, Paci S, et al. Reliability, cost-effectiveness, and safety of reuse of ancillary devices for ERCP. Gastrointest Endosc 2004;60:246-52.

14. London hospitals forced to cut millions from budgets, St. Joseph's dips into reserves. London (Ont.): AM980; 2015. Available: www.am980.ca /2015/03/31/42341/ (accessed 2015 July 20).

Affiliations: Department of Anesthesia and Perioperative Medicine (John-Baptiste, Martin); Department of Epidemiology and Biostatistics (John-Baptiste, Martin); Department of Otolaryngology-Head and Neck Surgery (Sowerby, Rotenberg), Interfaculty Program in Public Health (JOhn-Baptiste), Schulich School of Medicine and Dentistry, Western University; and Center for Medical Evidence, Decision Integrity, Clinical Impact (John-Baptiste, Martin), University of Toronto (Chin), Mount Sinai Hospital, Toronto, Ont.

Contributors: All of the authors contributed to the study design, collection and interpretation of the data, and drafting and revising of the manuscript. All of the authors approved of the final version to be published and agreed to act as guarantors of the work.

Funding: Ava John-Baptiste was funded by a start-up grant from the Department of Anesthesia and Perioperative Medicine.

Supplemental information: For reviewer comments and the original submission of this manuscript, please see www.cmajopen.ca/content/4/3/ E404/suppl/DC1 